\title{
Publishing: why and whom to?
}

\author{
Carlos Renato Zacharias (PhD)
}

Editor-in-Chief, International Journal of High Dilution Research

"To publish or to perish." This motto, inserted some years ago into academic culture, was co-responsible for the growth of many areas and groups of research. Underlying it, there is a stimulus to develop, spread and discuss research and scientific ideas. Thus, the ability to publish scientific papers has been used as an indicator of quality by agencies to promote and compare researchers. Is there anything wrong in developing research, new ideas, building hypotheses and then presenting them to a specialized community, for discussion, refinement and validation? The publication of an article in a peer-reviewed international journal is undoubtedly a certificate of quality, since it shows that original research was conducted, results were interpreted and made available to other researchers for criticism, correction and improvement.

Certainly the slogan "publish or perish" motivated many achievements and breakthroughs, but also stimulated some practices, say, addictions, which are leading the "good original intention" to a situation of degradation and disrepute. This model of evaluation is much discussed nowadays, and a new vocabulary has arisen to describe it: paperism, bibliographic Darwinism, sliced science, among others.

Focusing our reflection on homeopathy, we may ask: why to publish and who will be the beneficiaries? To answer these questions, it must be remembered that homeopathy was born out of an experimental program conducted outside more "official" paradigms and methods. This made its theoretical foundation inconsistent with scientific knowledge and practices as established nowadays, regardless of the quality and quantity of its clinical results.

To some extent, homeopathic clinical practice does not need scientific support to establish itself. In different times and places, it had moments of glory and also of disrepute. The latter usually emerged in the wake of questioning regarding its scientific foundations and possible mechanisms of action, coming from academic, political and commerciallyoriented settings. Glory, on the other hand, is related to its acceptance by users, its clinical results and as a reaction against conventional treatments, culminating in its official recognition in many countries.

So if academy is at the origin of criticism, why to adopt its methods and logic? Why to turn homeopathy into a science, developing research and publishing results within a vitiated structure? Moreover, what will be the benefit for the final users?

Let us analyze this problem more carefully. Each cycle of glory and disrepute happened in different cultural and scientific contexts. In ours, a radically wider and easier access to information translates into final users, e.g. potential patients, becoming more critical, requiring from doctors a position no longer dogmatic.

Researchers, although trained within rigid academicism, have already realized that there is a natural phenomenon, poorly described and still unexplained, involving high dilutions. This phenomenon is perceived not only in human clinics, but also in areas such as agronomy and veterinary. Environmentalists realize that this phenomenon may help in the recovery of the planet, and businesspeople foresee new products and services, with strong ecological appeal. Governments realize that they can reduce costs in the area of health, by adopting integrative and complementary therapies, which include homeopathy. But for all this to happen, quali and quantitative studies are needed

But... what phenomenon are we talking about? Biological reactions stimulated by homeopathic remedies?

Yes, but not only: the complete answer goes beyond homeopathic remedies. The process involving dilution together with agitation, or not, is not exclusive of homeopathy, but is also applied in isopathy, isoendopathy, hormesis, even in conventional pharmacology. On the other hand, the principle of similarity does not seem to be taken into account in many studies, even in clinical trials themselves.

Thus, there is a natural phenomenon, poorly described, with unknown mechanisms of action, a high unexplored potential revealed to us every day. 
This phenomenon has been named in different ways. We have named it here High Dilutions (HD) since it indirectly points out to the limits of Avogadro's number and cover virtually all forms of experimentation in this field.

Returning to our original question, we can answer that we need to publish to learn more about this phenomenon, because publication is the final result of extensive study, involving the questioning of paradigms, refinement of scientific methods, analysis of results, development of new hypotheses, shortly, serious research work. But this requires that publications have access to a diversified community of experts able to grasp, question and give feed-back regarding the phenomenon under consideration. In order for such a community to exist, we must invest in education into the various areas and fields of knowledge. Otherwise we will lose ourselves in a foolish and new dogma.

However, how to discuss education when we do not know the nature of the object of study? In fact, this kind of ignorance has never been an obstacle to the cultural and scientific development of humankind. Therefore, in the present situation, we need to be consistent and consequential in our reasoning, to be constantly aware of the accuracy of methods, without losing ourselves in their limitations or in sterile speculations, while at the same time trying to think in different ways. Finally, we have to write a new chapter in the history of culture and science. And for that, publication and discussion are essential.

If we desire knowledge to be built by different people in a highly diversified and dynamic society, we must have a readily available vehicle. Internet seems to be the most democratic and globalize vehicle at this beginning of the 21st century. However, economic and language barriers still need to be dealt with. Economic barriers may be removed by adopting public domain platforms and open systems for free information. Language barriers are harder to deal with, but not impossible if we work in cooperation.
For all these reasons, we are launching the International Journal of High Dilution Research (IJHDR) with the mission of being an electronic vehicle for wide and unrestricted access, specialized in the multidisciplinary field of high dilutions. The whole community, scientific or not, is invited to join us, as authors or readers, contributing to the process of peer-review performed by researchers and specialists, distributed all through the world. The requirement of quality can, thus, be met without violating freedom of expression or preventing new questions, assumptions and models to appear.

The International Journal of High Dilution Research is divided into thematic sections, covering basic research, pharmacology, clinics, sociological and historical research, as well specific sections dedicated to research in agronomy and veterinary. A special section is devoted to education, in order to build a common knowledge base, including experimental techniques, fundamental concepts, nomenclature, a discussion on the limitations and validity of models, in short, knowledge useful for all readers.

To summarize, in the field of research on high dilutions, publishing is fundamental, not in order to meet the quality indicators of scientific agencies, but to build together, step by step, the knowledge needed to establish the scientific basis of its object, which certainly will receive great attention in the 21st century. As a consequence, society as a whole will be benefited, due to direct application: in medicine, in agriculture, in animal care, environmental concerns and new technological developments.

On behalf of the Editorial Board of the International Journal of High Dilution Research, I would like to invite everyone to join us in this long but rewarding journey! 